\title{
THE MULTIPLICITY FUNCTION OF A LOCAL RING
}

BY

\author{
JAMES HORNELL
}

\begin{abstract}
Let $A$ be a local ring with maximal ideal $m$. Let $f \in A$, and define $\mu_{A}(f)$ to be the multiplicity of the $A$-module $A / A f$ with respect to $m$. Under suitable conditions $\mu_{A}(f g)=\mu_{A}(f)+\mu_{A}(g)$. The relationship of $\mu_{A}$ to reduction of $A$, normalization of $A$ and a quadratic transform of $A$ is studied. It is then shown that there are positive integers $n_{1}, \ldots, n_{s}$ and rank one discrete valuations $v_{1}, \ldots, v_{s}$ of $A$ centered at $m$ such that $\mu_{A}(f)=n_{1} v_{1}(f)+\cdots+n_{s} v_{s}(f)$ for all regular elements $f$ of $A$.
\end{abstract}

Let $A$ be a nonnull noetherian local ring with maximal ideal $m$. Let $d$ be the (Krull) dimension of $A$, the maximal length of a chain of prime ideals of $A$, excluding $A$. Let $k$ be the residue field $A / m$, and let $G_{m} A$ be the associated graded ring of $A$ with respect to $m$.

Let $f \in A$. If $A / A f$ is of dimension $d-1$ define $\mu_{A}(f)$ to be $e_{m}(A \mid A f)$, the multiplicity of the A-module $A / A f$ relative to $m$ in dimension $d-1[6, p . \mathrm{V}-2]$ or the multiplicity of the local ring $A / A f([7, p .294]$, or $[3, p .75])$. If $A / A f$ is of dimension $d$, define $\mu_{A}(f)$ to be $\infty$. Call $\mu_{A}(f)$ the multiplicity of $f($ at $m$ in $A$ ).

If $A$ is a regular local ring, $\mu_{A}$ is known to be the order valuation of $A$ [3, 40.2, p. 154]. If $A$ is entire $\mu_{A}(f g)=\mu_{A}(f)+\mu_{A}(g)$ (Proposition 1, §1). The order function $v_{A}$ of $A$ [7, p. 249] satisfies $v_{A}(f+g) \geqslant \min \left\{v_{A}(f), v_{A}(g)\right\}$, and (Proposition 2, §1) $v_{A}$ is a valuation if and only if $\mu_{A}$ is a multiple of $v_{A}$.

If the ideal (0) is unmixed in $A, \mu_{A}$ is found to extend to the components of $A$ (Lemma 2, $\S 2$ ). If $A$ is of dimension one, $\mu_{A}$ is found to extend to the normalization of $A$ (Lemma 3, §2). The extension of $A$ to the first neighborhood ring of $A$ (a quadratic transform of $A$ ) is found to preserve $\mu_{A}$ (Lemma 4, §3).

This is used to prove the theorem of $\S 4$, that there are positive integers $n_{1}, \ldots, n_{s}$ and discrete rank one valuations $v_{1}, \ldots, v_{s}$ of $A$ centered at $m$ such that for every regular element $f$ of $A$

$$
\mu_{A}(f)=n_{1} v_{1}(f)+\cdots+n_{s} v_{s}(f) .
$$

Received by the editors September 19, 1974.

AMS (MOS) subject classifications (1970). Primary 13H15; Secondary 13B20, 14 B05. 
The valuations $v_{1}, \ldots, v_{s}$ arise from (dimension one) normalization of the first neighborhood ring of $A$, and each $n_{i}$ is the product of the length of a primary component of (0) in $A$ of dimension $d$, the multiplicity of a $d$-dimensional component of the tangent cone of $A$ at the origin, the index of a normalization and another factor arising from a nonfinite normalization of an entire local ring of dimension one.

Let $p$ be a prime ideal of the noetherian ring $A$. The depth of $p$ will denote throughout the Krull dimension of $A / p$.

1. Elementary properties of $\mu_{A}$. For an $A$-module $M$ let $l_{A}(M)$ denote the length of $M$ as an $A$-module. If $p$ is a prime ideal of $A$ and if $\ell$ is an ideal of $A$ let $\lambda_{p}(\mathscr{V})=l_{A_{p}}\left(A_{p} / A_{p} \mathfrak{Z}\right)$.

Proposition 1. Let $f$ and $g$ be two elements of a local ring $A$, and assume either that $f$ is a regular element of $A$ or that $\mu_{A}(f)=\infty$. Then

$$
\mu_{A}(f g)=\mu_{A}(f)+\mu_{A}(g) .
$$

Proof. If $\mu_{A}(f)=\infty$, then $f$ and $f g$ are contained in a prime ideal of $A$ of depth $d$, and $\mu_{A}(f g)=\infty$.

Let $f$ be a regular element of $A$ and assume that $\mu_{A}(g)$ is finite. By [6, p. V-3], for any $h \in A$ such that $\mu_{A}(h)$ is finite,

$$
\mu_{A}(h)=\sum_{p} \lambda_{p}(A h) e_{m}(A / p)
$$

where the sum ranges over all prime ideals $p$ of $A$ of depth $d-1=\operatorname{dim} A-1$,

$$
0 \rightarrow A f \mid A f g \rightarrow A / A f g \rightarrow A / A f \rightarrow 0
$$

is exact, $A f / A f g \simeq A \mid A g$ as $A$-modules, $\lambda_{p}(A f g)=\lambda_{p}(A f)+\lambda_{p}(A g)$, and the proposition follows.

REMARK. Let $A=k[x, y]_{(x, y)}=k[X, Y]_{(X, Y)} /\left(X^{2}, X Y\right)$. By direct computation $\mu_{A}(y)=3$ and $\mu_{A}\left(y^{2}\right)=5$. Thus $\mu_{A}(f g)$ need not be $\mu_{A}(f)+$ $\mu_{A}(g)$ if neither $f$ nor $g$ is regular and if both $\mu_{A}(f)$ and $\mu_{A}(g)$ are finite.

Proposition 2. Let $A$ be an entire local ring and suppose the order function $v_{A}$ of $A$ is a valuation. Then

$$
\mu_{A}=e_{m}(A) v_{A} .
$$

Proof. $G_{m} A$ is entire, and if $f$ is a nonzero element of $A, f$ is superficial of degree $v_{A}(f)$. Thus [7, Lemma 4, p. 286], $\mu_{A}(f)=e_{m}(A / A f)=$ $e_{m}(A) \cdot v_{A}(f)$.

COROLlARY. If $A$ is a regular local ring then $\mu_{A}$ is the order valuation. 
REMARK. Let $A$ be an entire local ring of dimension one and suppose the order function $v_{A}$ of $A$ is a valuation. Then $G_{m} A$ is an entire graded ring over $k=A / m$ of dimension one which must be the polynomial ring in one variable over $k, \operatorname{dim}_{k} m / m^{2}=1, A$ is therefore a regular local ring, and $\mu_{A}=v_{A}$.

The following proposition gives a geometric definition of $\mu_{A}$. The local ring $A$ is said to be affine if it is the homomorphic image of a localization of a polynomial ring over a field.

Proposition 3. Let $A$ be an entire affine local ring which has an infinite residue field $k=A / m$. Then $A$ is the homomorphic image of an affine regular local ring $B$. Let $p$ be the kernel of this homomorphism of $B$ onto $A$, which is local, and notice that $B$ is equicharacteristic with residue field $k$. Let $d$ be the dimension of $A$. Then for every regular element $f$ of $A$,

$$
\mu_{A}(f)=\min _{f_{1}, \ldots, f_{d-1}}\left\{i\left(Z(B / p) \cdot Z\left(B / B f_{1}\right) \cdots Z\left(B / B f_{d-1}\right) \cdot Z(B / B f), m\right)\right\}
$$

where the minimum is taken over all $f_{1}, \ldots, f_{d-1} \in A$ for which the intersection is proper. For the definition and notation of the right-hand side of the equation see [1] and $[6, \S \mathrm{V}-\mathrm{C}]$.

REMARK. By applying Lemma $2, \S 2$ to $\mu_{A}(f)=e_{\left(f, f_{1}, \ldots, f d-1\right)}(A)$, by the additivity of $Z(B / p)$ and the linearity of $i(\cdot, m)$, the hypothesis that $A$ be entire may be dropped from Proposition 3.

REMARK. This proposition does not necessarily hold if the residue field is finite. For let $k$ be the field of $p^{n}$ elements, and let $A=k\left[X_{1}, X_{2}\right]_{\left(X_{1}, X_{2}\right)}$. Letting $\mu^{\prime}$ denote the formula of the right-hand side of the equality of the proposition, $\mu^{\prime}\left(X_{2}\left(\Pi_{\alpha \in k}\left(X_{1}-\alpha X_{2}\right)\right)\right)=p^{n}+2$, whereas $\mu_{A}\left(X_{2}\left(\Pi_{\alpha \in k}\left(X_{1}-\alpha X_{2}\right)\right)\right)=p^{n}+1$.

Proof of Proposition 3.

$$
\mu_{A}(f)=e_{\left(f_{1}, \ldots, f_{d-1}\right)}(A / A f)
$$

for some $f_{1}, \ldots, f_{d-1} \in m[7$, Theorem 22, p. 294]

$$
=\min _{f_{1}, \ldots, f_{d-1}}\left\{e_{\left(f_{1}, \ldots, f_{d-1}\right)}(A / A f)\right\}
$$

where $\left(f_{1}, \ldots, f_{d-1}\right)$ is an open ideal of $A / A f[7$, Lemma 2, p. 285]. The elements $f_{1}, \ldots, f_{d-1}$ have representatives in $B$ and in $A$, and consider $f_{1}, \ldots, f_{d-1}$ to be in either $B, A$ or $A / A f$.

Let $M$ be the maximal ideal of $B$, let $\hat{B}$ be the $M$-adic completion of $B$, and let $\hat{p}=\hat{B} p . \hat{A}=\hat{B} / \hat{p} . \hat{B} \simeq k\left[\left[X_{1}, \ldots, X_{n}\right]\right]$ for some $n$. Let $\left(f_{1}, \ldots, f_{d-1}\right)$ be an open ideal of $A / A f$. 


$$
e_{\left(f_{1}, \ldots, f_{d-1}\right)}(A / A f)=e_{\left(f_{1}, \ldots, f_{d-1}, f\right)}(A)
$$

$\left(\left[4\right.\right.$, p. 300] for $\left.\left((0):_{A} A f\right)=(0)\right)$

$$
\begin{aligned}
& =e_{\left(f_{1}, \ldots, f_{d-1}, f\right)}(\hat{B} / \hat{p}) \\
& =e_{\left(f_{1} \otimes 1, \ldots, f_{d-1} \otimes 1, f \otimes 1\right)} \\
& \quad\left(\left(\hat{B} \hat{\otimes}_{k} \hat{B} / \hat{p}\right) /\left(X_{1} \otimes 1-1 \otimes X_{1}, \ldots, X_{n} \otimes 1-1 \otimes X_{n}\right)\right) \\
& =e_{\left(X_{1} \otimes 1-1 \otimes X_{1}, \ldots, X_{n} \otimes 1-1 \otimes X_{n}, f_{1} \otimes 1, \ldots, f_{d-1} \otimes 1, f \otimes 1\right)}\left(\hat{B} \hat{\otimes}_{k} \hat{B} / \hat{p}\right)
\end{aligned}
$$

[4, p. 300], for $X_{1} \otimes 1-1 \otimes X_{1}, \ldots, X_{n} \otimes 1-1 \otimes X_{n}$ is a prime sequence in $\hat{B} \hat{\theta}_{k} \hat{B} / \hat{p}$ as will be shown below. As will also be shown below, $f_{1} \otimes 1$, $\ldots, f_{d-1} \otimes 1, f \otimes 1$ is a prime sequence in $\hat{B} \hat{\otimes}_{k} \hat{B} / \hat{p}$. The above equality may now be continued.

$$
\begin{aligned}
& e_{\left(f_{1}, \ldots, f_{d-1}\right)}(A / A f) \\
& =e_{\left(X_{1} \otimes 1-1 \otimes X_{1}, \ldots, X_{n} \otimes 1-1 \otimes X_{n}\right)}\left(\hat{B} /\left(f_{1}, \ldots, f_{d-1}, f\right) \hat{\otimes}_{k} \hat{B} / \hat{p}\right) \quad[4, \mathrm{p} .300] \\
& =\chi\left(B /\left(f_{1}, \ldots, f_{d-1}, f\right), B / p\right) \quad[6, \mathrm{p} . \mathrm{V}-12] \\
& =i\left(Z(B / p) \cdot Z\left(B / B f_{1}\right) \cdots Z\left(B / B f_{d-1}\right) \cdot Z(B / B f), m\right) \quad[6, \mathrm{p} . \mathrm{V}-20]
\end{aligned}
$$

It must be shown that $X_{1} \otimes 1-1 \otimes X_{1}, \ldots, X_{n} \otimes 1-1 \otimes X_{n}$ is a prime sequence in

$$
\left.\hat{B} \hat{\otimes}_{k} \hat{A} \simeq\left(\cdots\left(\hat{A}\left[\left[X_{1}\right]\right]\right)\left[\left[X_{2}\right]\right]\right) \cdots\right)\left[\left[X_{n}\right]\right] .
$$

By induction, it follows from the fact that $X_{1}-\alpha$ is a regular element of $R\left[\left[X_{1}\right]\right]$ for any $\alpha \in R$ where $R$ is a noetherian ring.

It must also be shown that $f \otimes 1, f_{1} \otimes 1, \ldots, f_{d-1} \otimes 1$ is a prime sequénce in $\hat{B} \hat{\otimes}_{k} \hat{A}$. $\left(f, f_{1}, \ldots, f_{d-1}\right)$ has height $d$ in $B$, so $f, f_{1}, \ldots, f_{d-1}$ is a prime sequence in $B$. Let $R$ and $S$ be two rings containing as a subring the field $k$, and let $\alpha$ be a regular element of $R$. $0 \rightarrow R \stackrel{m_{\alpha}}{\longrightarrow} R$ is exact where $m_{\alpha}$ denotes multiplication by $\alpha . S$ is $k$-flat, $0 \rightarrow R \otimes_{k} S \stackrel{m_{\alpha} \otimes_{k} S}{\longrightarrow} R \otimes_{k} S$ is exact, and $\alpha \otimes 1$ is a regular element of $R \otimes_{k} S$. It follows immediately that $f \otimes 1, f_{1} \otimes 1, \ldots, f_{d-1} \otimes 1$ is a prime sequence of $B \otimes_{k} A$. If $R$ is a Zariski ring and if $\hat{R}$ is the completion of $R$, then $f_{1}, \ldots, f_{d}$ is a prime sequence in $R$ if and only if $f_{1}, \ldots, f_{d}$ is a prime sequence in $\hat{R}$ [7, Chapter VIII, §5]. $A$ and $B$ are affine over $k$, so $B \otimes_{k} A$ is noetherian, and $B \otimes_{k} A$ is a Zariski ring with completion $\hat{B} \hat{\otimes}_{k} \hat{A}$. Thus $f \otimes 1, f_{1} \otimes 1, \ldots, f_{d-1} \otimes 1$ is a prime sequence in $\hat{B} \hat{\otimes}_{k} \hat{A}$.

2. The behavior of $\mu_{A}$ under reduction of $A$ and integral extension of $A$. Let $A$ be a nonimbedded local ring (the associated prime ideals of (0) in $A$ are all 
minimal). Let $I A$ be the integral closure of $A$ contained in $Q A$, the total quotient ring of $A$. The minimal (height zero) prime ideals of $A, I A$ and $Q A$ are in a bijective correspondence. Let $N$ be a minimal prime ideal of $A$. Then $\lambda_{N}(0)=$ $\lambda_{(I A) N}(0)=\lambda_{(Q A) N}(0)$, and $I(A / N) \simeq I A / I N$ where $I N=(I A) N . I A \simeq A_{1}^{\prime} \oplus$ $\cdots \oplus A_{n}^{\prime}$ where $I\left(A_{i}^{\prime}\right)=A_{i}^{\prime}$ and $A_{i}^{\prime}$ has a unique minimal prime ideal $N_{i}^{\prime}$.

$$
A_{1}^{\prime} \oplus \cdots \oplus A_{i-1}^{\prime} \oplus N_{i}^{\prime} \oplus A_{i+1}^{\prime} \oplus \cdots \oplus A_{n}^{\prime}=I N_{i}
$$

for $i=1, \ldots, n$ are the minimal prime ideals of $I A$. Thus a maximal ideal of $I A$ contains a unique minimal prime ideal.

LEMma 1. Let $A$ be a dimension one nonimbedded local ring with maximal ideal $m$. Let $I A$ be the integral closure of $A$ in its total quotient ring $Q A$. There are only a finite number of prime ideals $m_{1}, \ldots, m_{s}$ of $I A$ lying over $m$, and the indices $\left[I A / m_{i}: A / m\right]$ are finite for $i=1, \ldots, s$. Let $A_{i}=(I A)_{m_{i}}$. If $f$ is an element of $A$,

$$
l_{A}(A / A f)=\sum_{i=1, \ldots, s} n_{i} \lambda_{N_{i}}(0)\left[I A / m_{i}: A / m\right] l_{A_{i}}\left(A_{i} / A_{i} f\right)
$$

the $n_{i}$ being positive integers depending only upon $A / N$ where $N$ is the nil radical of $A$.

If $I A / I N$ is a noetherian $A$-module, then $n_{i}=1$ for $i=1, \ldots, s$. The $n_{i}$ may be greater than one, for in Nagata's example [3, E 3.2, p. 206], $s=1$ and $n_{1}=p$.

Proof. It may be assumed that $f$ is a regular element of $A$, for otherwise both sides of the equality are infinite. Let $B$ be a finite $A$-submodule of $I A$, and let $a \in A$ be regular and such that $a B \subset A$.

$$
\begin{aligned}
l_{A}(B / B f) & =l_{A}(B a \mid B a f)=l_{A}(A / A a f)-l_{A}(A / B a)-l_{A}(\text { Baf } / \text { Aaf }) \\
& =l_{A}(A / A f)+l_{A}(A \mid A a)-l_{A}(A / B a)-l_{A}(B a \mid A a) \\
& =l_{A}(A / A f) .
\end{aligned}
$$

By $[3$, Theorem 21.2, p. 70$]$, or by the first part of the proof of $[7$, Theorem 24, p. 297],

$$
l_{A}(A / A f)=\sum_{i=1, \ldots, s_{B}}\left[B / p_{i}: A / m\right] l_{B}\left(B_{p_{i}} / B_{p_{i}} f\right)
$$

where $p_{1}, \ldots, p_{s_{B}}$ are the prime ideals of $B$ lying over $m$. There are a finite number of prime ideals in $I A$ lying over $m$, for $s_{B} \leqslant l_{A}(A / A f)$. Let $m_{1}, \ldots$, $m_{s}$ be the maximal ideals of $I A$. Note that

$$
l_{A}\left(\operatorname{dir} \lim _{\iota} M_{\iota}\right) \leqslant \max _{\iota}\left\{l_{A}\left(M_{\iota}\right)\right\}
$$

$I A / m_{i}=\operatorname{dir} \lim _{B} B / B \cap m_{i}$ and $\left[I A / m_{i}: A / m\right]$ is finite. 
Let $\alpha_{i} \in I A$ be such that $\alpha_{i} \in m_{i}$ and $\alpha_{i} \notin \bigcup_{j \neq i} m_{j}$. Let $\beta_{1}, \ldots, \beta_{t} \in I A$ be such that

$$
\left[A\left[\beta_{1}, \ldots, \beta_{t}\right] /\left(m_{i} \cap A\left[\beta_{1}, \ldots, \beta_{t}\right]\right): A / m\right]=\left[I A / m_{i}: A / m\right]
$$

for $i=1, \ldots, s$. Let $A^{\prime}=A\left[\alpha_{1}, \ldots, \alpha_{s}, \beta_{1}, \ldots, \beta_{t}\right]$. By the formula above, letting $A$ be $A_{A^{\prime} \cap_{m} m_{i}}$, it can be assumed that $s=1$ and $\left[I A / m_{i}: A / m\right]=1$. Then for a finite extension $B \subset I A$ of $A, l_{A}(A \mid A f)=l_{B}(B / B f)$. The nil radical $N$ of $A$ is now a prime ideal.

First assume that $I(A / N)$ is a noetherian $A / N$-module. By a finite extension of $A$ in $I A$ it can be assumed that $A / N$ is normal, and thus that $A / N$ is a regular local ring of dimension one [3, Theorem 33.2, p. 115 and Theorem 21.4, p. 40]. Let $x \in m / N$ generate $m / N$ in $A / N$. Let

$$
(0)=N_{0} \subset N_{1} \subset \cdots \subset N_{t-1}=N A_{N} \subset N_{t}=A_{N}
$$

be a composition series of $A_{N}$ over $A_{N}$, and let $n_{i}=A \cap N_{i} \cdot n_{i} / n_{i-1}$ is a principal $A / N$-module: If $\alpha_{1}, \ldots, \alpha_{q} \in n_{i} / n_{i-1}$ are nonzero and generate $n_{i} / n_{i-1}$ as an $A$ or $A / N$-module, there are $v, v_{j} \in A \sim N$ such that $v_{j} \alpha_{j}=v \alpha_{1}$ for $j=1, \ldots, q$ (for there is a bijective correspondence between the ideals of $A_{N}$ and their contractions in $A$ ). Viewed as $A / N$-modules, $\alpha_{j}=u_{j} x^{t_{j}} \alpha_{1}$ where $u_{j}$ is a unit in $A / N$ and where $t_{j}$ is an integer. Let $t_{k}=\min \left\{t_{1}, \ldots, t_{q}\right\}$. $n_{i} / n_{i-1}=A \alpha_{k}$. So there are $a_{1}, \ldots, a_{t} \in N$ with $n_{i}=\left(a_{1}, \ldots, a_{i}\right)$. For $i=1, \ldots, t$,

$$
0 \rightarrow \frac{n_{i}+A f}{n_{i-1}+A f} \rightarrow \frac{A}{n_{i-1}+A f} \rightarrow \frac{A}{n_{i}+A f} \rightarrow 0
$$

is exact. Map $A \rightarrow\left(n_{i}+A f\right) /\left(n_{i-1}+A f\right)$ by $y \mapsto y a_{i}+\left(f, a_{1}, \ldots, a_{i-1}\right)$. Suppose $y a_{i} \in\left(f, a_{1}, \ldots, a_{i-1}\right)$. There are $c, c_{1}, \ldots, c_{i-1} \in A$ such that $c f=c_{1} a_{1}+\cdots+c_{i-1} a_{i-1}-y a_{i} \cdot y \notin N$ and $n_{i}$ is $N$-primary because it is the contraction of an $A_{N} N$-primary ideal, so $c \in\left(a_{1}, \ldots, a_{i}\right)$. Thus there is an element $b$ of $A$ such that $y a_{i}-b a_{i} f \in\left(a_{1}, \ldots, a_{i-1}\right) . a_{i} \notin\left(a_{1}, \ldots, a_{i-1}\right)$ which is $N$-primary, so $y-b f \in N$. Hence

$$
\left(n_{i}+A f\right) /\left(n_{i-1}+A f\right) \simeq A /(N+A f),
$$

and

$$
l_{A}(A / A f)=\lambda_{N}(0) l_{A / N}(A /(N+A f))=\lambda_{N}(0) l_{I A / I N}(I A / I A \cdot f) .
$$

Now drop the assumption that $I(A / N)$ is a finite $A / N$-module. Let $\hat{A}$ be the $m$-adic completion of $A . l_{A}(A / A f)=l_{\hat{A}}(\hat{A} \mid \hat{A} f)$. The pair $A, m$ is a Zariski ring, so $(A / N)^{\wedge} \simeq \hat{A} / \hat{N}, \hat{A}$ and $\hat{N}$ are unmixed [7, Chapter VIII, $\left.\S 4\right]$. Letting $M_{j}$ be a minimal prime ideal of $\hat{A}, I\left(\hat{A} / M_{j}\right)$ is a finite $\hat{A} / M_{j}$-module [3, Theorem 32.1, p. 112]. By the finite case above 


$$
l_{\hat{A}}(\hat{A} \mid \hat{A} f)=\sum_{j} \lambda_{M_{j}}(0) l_{\hat{A} / M_{j}}\left(\left(\hat{A} / M_{j}\right) /\left(\hat{A} / M_{j}\right) f\right) .
$$

$A \subset A_{N} \subset \hat{A}_{M_{j}}$ canonically. Let

$$
\text { (0) }=N_{0} \subset N_{1} \subset \cdots \subset N_{t-1}=A_{N} N \subset N_{t}=A_{N}
$$

be a composition series of $A_{N} \cdot N_{i} \otimes_{A_{N}} \hat{A}_{M_{j}}$ can be refined into a composition series for $A_{M_{j}}$. Now $N_{i} / N_{i-1} \simeq A_{N} / A_{N} N$, this completion and localization are exact, so $N_{i} / N_{i-1} \otimes_{A_{N}} A_{M_{j}}$ are all isomorphic for $i=1, \ldots, t$ of length

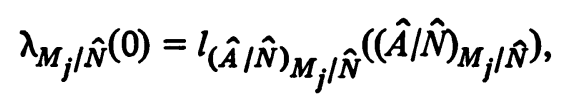

and $\lambda_{M_{j}}(0)=\lambda_{N}(0) \lambda_{M_{j} / \hat{N}}(0)$. Thus

$$
l_{\hat{A}}(\hat{A} / \hat{A} f)=\lambda_{N}(0) l_{\hat{A} / \hat{N}}((\hat{A} \mid \hat{N}) /(\hat{A} / \hat{N}) f),
$$

and it follows that

$$
l_{A}(A / A f)=\lambda_{N}(0) l_{A / N}(A /(N+A f)) .
$$

$I(A / N) \simeq I A / I N$, and $I A / I N$ is a regular local ring of dimension one [3, Theorem 33.2, p. 115 and Theorem 12.4, p. 40]. Let $x$ be a generator of the maximal ideal $m_{1}$ of $I A$ and let $u$ be a unit in $I A$ such that for some integer $n$, $f=u x^{n}$. By a finite extension of $A$ it may be assumed that $u$ and $x$ are elements of $A$. To finish the proof, notice that $l_{I A}(I A /(I A) x)=1$ and $I N \subset(I A) x$ so that

$$
\frac{l_{A / N}((A / N) /(A / N) f)}{l_{I A}(I A /(I A) f)}=l_{A / N}((A / N) /(A / N) x) .
$$

Let $n_{1}=l_{A / N}((A / N) /(A / N) x)$.

LEMMA 2. Let $A$ be a local ring with maximal ideal $m$, let $N_{1}, \ldots, N_{n}$ be the prime ideals of $A$ of depth $d=\operatorname{dim} A$. For every regular element $f$ of $A$

$$
\mu_{A}(f)=\sum_{i=1, \ldots, n} \lambda_{N_{i}}(0) \mu_{A / N_{i}}\left(f+N_{i}\right) .
$$

Proof. If $\operatorname{dim} A=0$, the formula holds trivially. Let $p$ be a prime ideal of $A$ of depth $d-1$ and containing $f$. Then $B=A_{p}$ is of dimension one and is nonimbedded, for $f$ is a regular element. Note that if $N_{i} \subset p$, then $\lambda_{N_{i}}(0)=\lambda_{B N_{i}}(0)$. By Lemma 1 , applied to $B$ and to $B / B N_{i}$ for $N_{i} \subset p$,

$$
l_{B}(B / B f)=\sum_{N_{i} \subset p} \lambda_{N_{i}}(0) l_{B / B N_{i}}\left(\left(B \mid B N_{i}\right) /\left(B / B N_{i}\right) f\right),
$$

and by $[6, p . V-3]$, 


$$
\begin{aligned}
\mu_{A}(f) & =\sum_{p} l_{p}(A / A f) e_{m}(A / p) \\
& =\sum_{p} \sum_{N_{i} \subset p} \lambda_{N_{i}}(0) l_{p / N_{i}}\left(\left(A / N_{i}\right) /\left(A / N_{i}\right) f\right) e_{m}(A / p) \\
& =\sum_{i=1, \ldots, n} \lambda_{N_{i}}(0) \mu_{A / N_{i}}\left(f+N_{i}\right) .
\end{aligned}
$$

LEMMA 3. Let $A$ be a dimension one local ring with maximal ideal $m$, let $m_{1}, \ldots, m_{s}$ be the prime ideals of $I A$ lying over $m$, and let $A_{i}=I A_{m_{i}}$ For every regular element $f$ of $A$,

$$
\mu_{A}(f)=\sum_{i=1, \ldots, s} \lambda_{N_{i}}(0) n_{i}\left[I A / m_{i}: A / m\right] \mu_{A_{i}}(f)
$$

for some positive integers $n_{1}, \ldots, n_{s}$ where $N_{i}$ is the minimal prime ideal of $A_{i}$.

This is a restatement of Lemma 1 . (If $A$ is imbedded, the only regular elements of $A$ are the units, and the formula holds trivially.)

REMARK. Lemma 3 does not necessarily hold if the dimension of $A$ is greater than one. Let

$$
A=k[w, x, y, z]_{(w, x, y, z)}=k[W, X, Y, Z]_{(W, X, Y, Z)} /\left(X^{2}-Z^{3}, X Y-W^{3}\right)
$$

where $k$ is a field. By direct computation $\mu_{A}(x)=9$ and $\mu_{A}(y)=6$.

$$
A \simeq k\left[t s, t^{3}, s^{3}, t^{2}\right]_{\left(t s, t^{3}, s^{3}, t^{2}\right)} \subset k[s, t]_{(s, t)}
$$

where $s$ and $t$ are independent transcendentals over $k$, and $I A \simeq k[s, t]_{(s, t)}$. Thus $\mu_{I A}(x)=\mu_{I A}(y)=3$. By the Corollary of Proposition $2, \mu_{I A}=v$ where $v$ is the order valuation of $k[s, t]_{(s, t)}$ having valuation ring $k(s / t)[t]_{(t)} \cdot \mu_{A}=$ $v+w$ where $w$ is the valuation having valuation ring $k\left(t / s^{2}\right)[s]_{(s)}$. (See §4.)

3. The first neighborhood ring of $A$ : a quadratic transform of $A$ which is compatible with $\mu_{A}$. Let $G_{m} A$ be the associated graded ring of $A$ with respect to $m$. Let $m=\left(x_{1}, \ldots, x_{n}\right)$. The natural homomorphisms

$$
A\left[X_{1}, \ldots, X_{n}\right] \rightarrow k\left[X_{1}, \ldots, X_{n}\right] \rightarrow G_{m} A
$$

(where $k=A / m$ ) will be used. Let $A[X]$ denote $A\left[X_{1}, \ldots, X_{n}\right]$, and let $k[X]$ denote $k\left[X_{1}, \ldots, X_{n}\right] . I$ will denote the ideal $\left(X_{1}, \ldots, X_{n}\right)$ of $A[X]$, $k[X]$, and $G_{m} A$.

A familiarity with Northcott's The neighborhoods of a local ring [5] is assumed. For the definition of the first neighborhood ring $\$$ of $A$, see [5, p. 361]. Let $p_{1}, \ldots, p_{r}$ be the height one prime ideals of $₫$ lying over $m$, and let $p_{i}$ be the prime ideal of $G_{m} A$ corresponding to $\mathfrak{p}_{i}$ [5, Propositions 1-4]. The preimage of $p_{i}$ in $k[X]$ will also be denoted by $p_{i}$. For the definition of a superficial element of $A$ see [5, p. 362], [3, p. $7 \dot{2}$ and Theorem 30.1, p. 103], or $[7$, p. 285]. 
Lemma 4. Let $A$ be an entire local ring with maximal ideal $m$ and an infinite residue field $k$. Let $\Re$ be the first neighborhood ring of $A$, let $p_{1}, \ldots$,

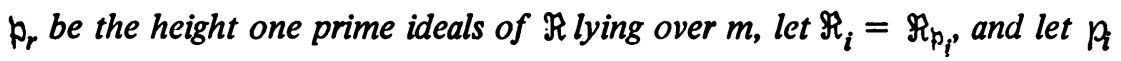
be the prime ideal of $G_{m} A$ corresponding to $p_{i}$. Then

$$
\mu_{A}(f)=e_{I}\left(G_{m} A / p_{1}\right) \mu_{\Re_{1}}(f)+\cdots+e_{I}\left(G_{m} A / p_{r}\right) \mu_{\Re_{r}}(f)
$$

for all $f \in A$.

Proof. The equality is easily shown to hold for a superficial element of $A$. Let $f \in A$ be superficial of degree s. $\mu_{A}(f)=e_{m}(A / A f)=\operatorname{se}_{m}(A)[7$, Lemma 4, p. 286], and

$$
\mu_{A}(f)=s\left(e_{I}\left(k[X] / p_{1}\right) e_{p_{1}}\left(\Re_{1} / \Re_{1} m\right)+\cdots+e_{I}\left(k[X] / p_{r}\right) e_{p_{r}}\left(\Re_{r} / \Re_{r} m\right)\right)
$$

[5, formula E, p. 370]. Let $x$ be a superficial element of $A$ of degree one. $f / x^{s}$ $\in \Re_{i}, \Re_{i} m=\Re_{i} x$ for $i=1, \ldots, r$, and

$$
\begin{aligned}
\mu_{A}(f) & =s\left(e_{I}\left(k[X] / p_{1}\right) \mu_{\Re_{1}}(x)+\cdots+e_{I}\left(k[X] / p_{r}\right) \mu_{\Re_{r}}(x)\right) \\
& =e_{I}\left(k[X] / p_{1}\right) \mu_{\Re_{1}}(f)+\cdots+e_{I}\left(k[X] / p_{r}\right) \mu_{\Re_{r}}(f) .
\end{aligned}
$$

The proof of the equality in general will occupy the rest of this section.

First let $\operatorname{dim} A \geqslant 2$. The proof will proceed by fixing the element $f \in A$ and blowing up $A$ to a one-dimensional ring $B$ such that $\mathfrak{\Re}^{1}=\Re_{1} \cap \cdots \cap R_{r}$ is an integral extension of $B$ and such that $G_{m B}(B / B f)$ is nearly a linear section of $G_{m}(A / A f)$.

Let $v_{A}$ be the order function of $A$ with respect to $m$. Let $x$ be a superficial element of $A$ of degree one, let $m=\left(x_{1}, \ldots, x_{n}\right)$ and let $\Pi$ be a form of degree one in $A\left[X_{1}, \ldots, X_{n}\right]$ with $x=\Pi\left(x_{1}, \ldots, x_{n}\right)$. $\Pi$ will also denote its image modulo $m$ in $k\left[X_{1}, \ldots, X_{n}\right]$. Consider the diagram,

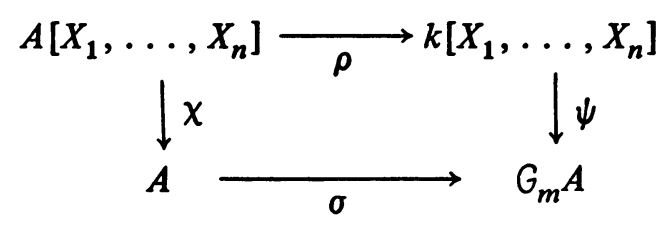

where $\sigma(g)=\left(g+m^{v}{ }^{(g)+1}\right) / m^{v_{A}}(g)+1, \psi$ is the canonical homomorphism and $k=A / m, \chi$ is the homomorphism with $\chi\left(X_{i}\right)=x_{i}$ and $\left.\chi\right|_{A}=\mathrm{id}_{A}$, and $\rho(F)$ is the leading form modulo $m$ of $F . \sigma(A f)$ is an ideal of $G_{m} A$, but $\sigma$ need not be a homomorphism. Let $\tau A f=\psi^{-1} \sigma(A f)$, let $\omega A f=\chi^{-1}(A f)=\left(X_{1}-x_{1}, \ldots\right.$, $\left.X_{n}-x_{n}, f\right)$, and let $\sigma A f$ denote $\sigma(A f)$.

$\rho(\omega A f)=\tau A f$. First notice that if $E \in \omega A f$ and $\operatorname{deg} E=v_{A}(\chi E)=s$ then $\psi \rho E=\psi\left(E+m[X]+I^{s+1}\right)=E\left(x_{1}, \ldots, x_{n}\right)+m^{s+1}$. Secondly notice that $\psi^{-1}(0)=\tau A 0 \subset \rho(\omega A f)$. If $E \in \omega A f$ and if $\psi \rho E=0$ then $\rho E \in \psi^{-1}(0) \subset$ 
$\rho(\omega A f)$. If $E \in \omega A f$ and if $\psi \rho E \neq 0$ then $\operatorname{deg} E=v_{A}(\chi E), \psi \rho E=\sigma \chi E$, and $\rho E \in \tau A F$. Hence $\rho(\omega A f) \subset \tau A f$. Let $e \in A f$. Let $E \in \omega A f$ be such that $\operatorname{deg} E=v_{A}(e)$ and $\chi E=e$. Then $\sigma e=\psi \rho E, \rho E \in \psi^{-1}(\sigma e)$, and $\tau A f \subset \rho(\omega A f)$.

Let $p$ be an isolated prime ideal of $\tau A 0$. Then depth $p=\operatorname{dim} A-$ height $p$ $\geqslant 2$ and $\operatorname{depth}(p, \Pi) \geqslant 1$.

Choose $\Theta$ to be a form of degree one in $A[X]=A\left[X_{1}, \ldots, X_{n}\right]$ such that $y=\Theta\left(x_{i}\right)$ is a superficial element of $A$ and a superficial element of $A \mid A f$, such that $\Theta$ is contained in no isolated prime ideal of $(p, \Pi)$ for any isolated prime ideal $p$ of $\tau A 0$, and such that $y$ is contained in no associated prime ideal of $A x$ other than possibly $m$. Each condition is viewed as a condition on form ideals in $k[X]$. Let $\Theta$ also denote its image modulo $m$ in $k[X]$.

Let $u=y / x$. Let $P$ be the kernel of the canonical homomorphism of $A[U]$ onto $A[u]$ where $A[U]$ is the polynomial ring in one variable and $U$ maps to $u$. $P \cap A=(0)$, and it follows that $P$ is of height one in $A[U]$. Letting $D_{A}$ denote the set of prime ideals of $A$ which occur as an imbedded prime ideal of a proper principal ideal of $A$ (see [2, §6]), $Q \in D_{A}[U]$ if and only if $Q \cap A \in D_{A}$ and $Q=(Q \cap A) \cdot A[U], y-x U$ is prime in $A[U]$ if and only if $x, y$ form a prime sequence in $A$, but this is the case if and only if $m \notin D_{A}$. If $m \notin D_{A}$ then $P=$ $(y-x U)$, and $P \subset m[U]$. If $m \in D_{A}$ then $P$ and $m[U]$ are the associated prime ideals of $(y-x U)$. For if $Q$ is an associated prime ideal of $(y-x U)$ of height greater than one then $x, y \in Q \cap A$ and $Q=m[U]$. If $Q$ is of height one, either $Q \cap A=q \neq(0)$, in which case $Q=q[U]$ and $x, y \in q$ which contradicts the choice of $y$, or $Q \cap A=(0)$ in which case $Q=(Q A)[U] \cdot(y-x U)=P$. It again follows that $P \subset m[U]$. So $A[u] / m[u] \simeq k[U]$, and $\bar{u}=u+m \cdot A[u]$ is transcendental over $k$.

Let $S=A[u] \sim m A[u]$ and let $B=S^{-1} A[u] . B / m B \simeq k(\bar{u})$ a simple transcendental extension of $k$. $\operatorname{Dim} A[U]=\operatorname{dim} A+1$, the kernel $P$ of the homomorphism $A[U] \rightarrow A[u]$ is height one, $m[U]$ is of height equal to $\operatorname{dim} A$, and $\operatorname{dim} B=\operatorname{dim} A-1$. Consider $G_{m B} B$ and the commutative diagram

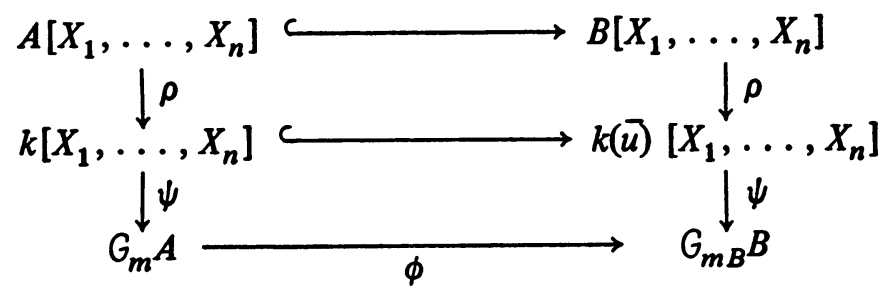

where $\phi$ is the canonical homomorphism induced by the inclusion $A \subset B$. Define $\sigma, \tau$ and $\omega$ for $B$ as was done for $A$. Notice that $\omega A f \subset \omega B f$, so $\tau A f \subset \tau B f$. $\Theta$ $-u \Pi \in \omega B f$. Let $q$ be an associated prime ideal of $\tau A f$ which is not $I=\left(X_{1}\right.$, $\left.\ldots, X_{n}\right)$. If $\Theta-\bar{u} \Pi \in k(\bar{u}) \cdot q$, then $\Theta-\bar{u} \Pi \in k[\bar{u}] \cdot q$ and $\Theta \in q$, which is 
a contradiction to the superficiality of $y$. Therefore $\Theta-\bar{u} \Pi \notin k(\bar{u}) q$, and $\Theta-$ $\bar{u} \Pi$ is superficial as an element of $k(\bar{u})[X] / k(\bar{u}) \cdot \tau A f$.

Now $\mu_{A}(f)=e_{I}(k[X] / \tau A f)$ and $\mu_{B}(f)=e_{I}(k(u)[X] / \tau B f)$. These modules are homogeneous and their lengths over $k[X]$ or $k(u)[X]$ are their dimensions over $k$ or $k(\bar{u})$. Thus $\mu_{A}(f)=e_{I}(k(\bar{u})[X] / k(\bar{u}) \cdot \tau A f)$. By Lemmas 3 and 4 of $[7$, pp. 285-286], if $\operatorname{dim} A>2$,

$$
e_{I}(k(\bar{u})[X] / k(\bar{u}) \cdot \tau A f)=e_{I}(k(\bar{u})[X] /(\tau A f, \Theta-\bar{u} \Pi)),
$$

and if $\operatorname{dim} A=2$,

$$
\begin{aligned}
e_{I}(k(\bar{u})[X] / k(\bar{u}) \cdot \tau A f)= & e_{I}(k(\bar{u})[X] /(\tau A f, \Theta-\bar{u} \Pi)) \\
& -l_{k(\bar{u})[X]}\left(I^{c}+\left(\left(I^{n}, \tau A f\right): \Theta-\bar{u} \Pi\right) /\left(I^{c}, \tau A f\right)\right)
\end{aligned}
$$

for all large enough $n$ and $c$ with $n>c$. Because $\Theta-\bar{u} \Pi$ is contained in no associated prime ideal of $k(\bar{u}) \cdot \tau A f$ other than possibly $I$, the homogeneous parts of like degree of $k(\bar{u}) \cdot \tau A f$ and of $(k(\bar{u}) \cdot \tau A f: \Theta-\bar{u} \Pi)$ are equal for sufficiently large degree. So for large enough $n$ and $c$, over $k(\bar{u})$

$$
\left(I^{c}+\left(\left(I^{n}, \tau A f\right): \Theta-\bar{u} \Pi\right) /\left(I^{c}, \tau A f\right)\right) \simeq(k(\bar{u}) \cdot \tau A f: \Theta-\bar{u} \Pi) / k(\bar{u}) \cdot \tau A f
$$

and for $\operatorname{dim} A=2$,

$$
\begin{aligned}
e_{I}(k(\bar{u})[X] / k(\bar{u}) \cdot \tau A f)= & e_{I}(k(\bar{u})[X] /(\tau A f, \Theta-\bar{u} \Pi)) \\
& -\operatorname{dim}_{k(\bar{u})}(k(\bar{u}) \cdot \tau A f: \Theta-\bar{u} \Pi) / k(\bar{u}) \cdot \tau A f .
\end{aligned}
$$

Let

$$
\alpha=\operatorname{dim}_{k(\bar{u})} \tau B f /(\tau A f, \Theta-\bar{u} \Pi)
$$

and

$$
\beta=\operatorname{dim}_{k(\bar{u})}(k(\bar{u}) \cdot \tau A f: \Theta-\bar{u} \Pi) / k(\bar{u}) \cdot \tau A f .
$$

It is to be shown that $\alpha=\beta$. Then $\alpha$ is finite, for $\beta$ is finite by the superficiality of $\Theta-\bar{u} \Pi$, and it follows that if $\operatorname{dim} A>2, \mu_{A}(f)=\mu_{B}(f) . \operatorname{If} \operatorname{dim} A=2$ it follows from $\alpha=\beta$ that $\mu_{A}(f)=\mu_{B}(f)$.

If $\mathscr{Q}$ is a set of polynomials in $X_{1}, \ldots, X_{n}$, let $\mathscr{U}_{(d)}$ be the set of all elements of $\mathfrak{U}$ which have no nonzero homogeneous component of degree strictly less than $d$, and let $\mathscr{U}_{d}$ be the set of all homogeneous elements of $\mathcal{U}$ of degree $d$.

Let $S=A[U] \sim m[U]$, and let $A(U)$ denote $S^{-1} A[U]$. Let $\tau(P, f)=$ $\rho(P, \omega A(U) f)$ and $\tau(\Theta-U \Pi, f)=\rho(\Theta-U \Pi, \omega A(U) f)$. Consider

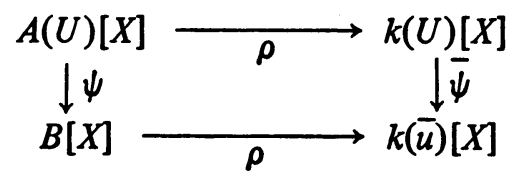


where $\rho(\alpha)$ is the leading form in $X_{1}, \ldots, X_{n}$ of $\alpha$ modulo $m A(U)[X]$ or $m B[X]$, where $\psi(U)=u$ and $\left.\psi\right|_{A[X]}=\mathrm{id}_{A[X]}$, and where $\bar{\psi}(U)=\bar{u}$ and $\left.\bar{\psi}\right|_{k[X]}=\mathrm{id}_{k[X]}$. Because $P \subset(P, \omega A(U) f)$,

$$
\bar{\psi} \tau(P, f)=\rho \psi(P, \omega A(U) f)=\tau B f .
$$

Note that $\bar{\psi}: k(U)[X] \rightarrow k(\bar{u})[X]$ is an isomorphism over the isomorphism $k(U) \simeq k(\bar{u})$ induced by $\bar{\psi}$. Let

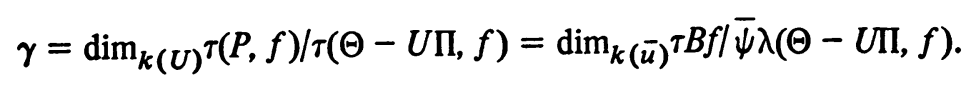

Then

$$
\operatorname{dim}_{k(U)} \tau(f, \Theta-U \Pi) /(\tau A f, \Theta-U \Pi)=\alpha-\gamma .
$$

Let $H$ be $\rho\left((\omega A(U) f)^{\wedge}:{ }_{A(U)[[X]\}} \Theta-U I I\right)$ where ^ denotes the $I$-adic completion. Let $Q$ be an associated prime ideal of $\omega A(U) f$. $\left(X_{1}-x_{1}, \ldots\right.$, $\left.X_{n}-x_{n}\right) \subset Q$, so $Q \subset(m A(U), I) . A(U)[X]_{(m A(U), I)}$ with the $I$-adic topology is a Zariski ring with completion $A(U)[[X]]$. Hence

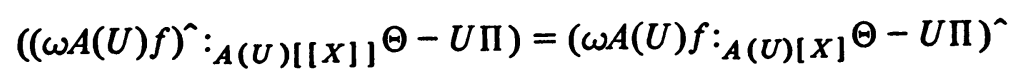

[7, Corollary 4, p. 266], and $H=p(\omega A(U) f: \Theta-U \Pi)$. So $\bar{\psi} H \subset(k(\bar{u}) \cdot \tau A f$ : $\Theta-U \Pi)$. Let

$$
\delta=\operatorname{dim}_{k(U)} H / k(U) \cdot \tau A f .
$$

Then

$$
\operatorname{dim}_{k(U)}(k(U) \cdot \tau A f: \Theta-U \Pi) / H=\beta-\delta .
$$

It is to be first shown that $\alpha-\gamma=\beta-\delta$.

Let $M \in A(U)\left[X_{1}, \ldots, X_{n}\right]$ be homogeneous of degree $d$ such that $M+$ $m A(U)[X] \in \tau(\Theta-U \Pi, f)$. The following four assertions follow easily from the fact that $x_{i}-X_{i} \in \omega A(U) f$. There is an integer $h \leqslant d-1$ and forms $H_{i} \in$ $A(U)[X]$ of degree $i=h, \ldots, d-1$ such that

$$
(\Theta-U \Pi)\left(H_{h}+\cdots+H_{d-1}\right)+M \in \omega A(U) f+A(U)[X]_{(d+1)} .
$$

If $M-M^{\prime} \in m A(U)[X]_{d}$, then

$$
(\Theta-U \Pi)\left(H_{h}+\cdots+H_{d-1}\right)+M^{\prime} \in \omega A(U) f+A(U)[X]_{(d+1)} .
$$

If $H_{h}-H_{h}^{\prime} \in m A(U)[X]_{h}$, there are forms $H_{i}^{\prime} \in A(U)[X]$ for $i=h+1, \ldots$, $d-1$ such that

$$
(\Theta-U \Pi)\left(H_{h}^{\prime}+\cdots+H_{d-1}^{\prime}\right)+M \in \omega A(U) f+A(U)[X]_{(d+1)} .
$$

If $F \in A(U)[X]_{d}$ and if $F+m A(U)[X] \in k[X] \cdot \tau A f$, then 


$$
(\Theta-U \Pi)\left(H_{h}+\cdots+H_{d-1}\right)+(M+F) \in \omega A(U)[X]+A(U)[X]_{(d+1)} .
$$

Note that $H_{h}+m A(U)[X] \in(k(U) \cdot \tau A f: \Theta-U I)$. Let $h(M)<\operatorname{deg} M$ be the maximal degree of all such $H_{h}$ as above. Let $H(M)$ be the set of all such $H_{n}$ as above with $h=h(M) . M+m A(U)[X] \in(\tau A f, \Theta-U I I)$ if and only if $h(M)=$ $\operatorname{deg} M-1$ which is true if and only if $\|_{h(M)} \subset H(M)$ (which in this case is $\left.A(U)[X]_{n(M)}\right)$. If $b \in A(U) \sim m A(U), b H(M)=H(b M)$. If $H \in H(M)$ then

$$
\left(H+m A(U)[X]_{h(M)}\right)+H_{h(M)} \subset H(M) / m A(U)[X]_{h(M)},
$$

and $H(M)$ will be considered as a subset of $(k(U) \cdot \tau A f: \Theta-U T) / i l$.

A $k(U)$-linear injection of $\tau(f, \Theta-U \Pi) /(\tau A f, \Theta-U \Pi)$ into $(k(U) \cdot \tau A f: \Theta-U \Pi) / H$ is to be defined. Let $M_{1}, \ldots, M_{a} \in A(U)[X]$ be forms such that their residues modulo $m A(U)[X]$ are in $\tau(f, \Theta-U I I)$, such that their residues in $\tau(f, \Theta-U \Pi) /(\tau A f, \Theta-U \Pi)$ are linearly independent over $k(U)$, such that $h\left(M_{i}\right) \leqslant h\left(M_{i+1}\right)$ and such that if $h\left(M_{i}\right)=h\left(M_{i+1}\right)$ then $\operatorname{deg} M_{i} \geqslant$ $\operatorname{deg} M_{i+1}$. Choose $\eta_{i} \in H\left(M_{i}\right)$. Suppose $\eta_{i}, \ldots, \eta_{t-1}$ are linearly independent over $k(U)$, and suppose $\eta_{t}=\bar{\alpha}_{1} \eta_{1}+\cdots+\bar{\alpha}_{t-1} \eta_{t-1}$ where $\alpha_{i} \in A(U)$. The $\bar{\alpha}_{i}$ are nonzero only for those $M_{i}$ with $h\left(M_{i}\right)=h\left(M_{t}\right) . h\left(M_{t}\right)=h\left(M_{t-1}\right)$, for $\eta_{t} \neq 0$. Let $M_{s}, \ldots, M_{t-1}$ be exactly those $M_{i}$ with $i<t, h\left(M_{i}\right)=h\left(M_{t}\right)$ and $\operatorname{deg} M_{i}=\operatorname{deg} M_{t}$. Then $h\left(M_{t}-\alpha_{s} M_{s}-\cdots-\alpha_{t-1} M_{t-1}\right)>h\left(M_{t}\right)$, so replace $M_{t}$ by $M_{t}-\alpha_{s} M_{s}-\cdots-\alpha_{t-1} M_{t-1}$, choose a new $\eta_{t}$, and reorder $M_{t}, \ldots, M_{a}$. With a finite number of repetitions of the above process $\eta_{1}, \ldots, \eta_{t}$ will be linearly independent, for at worst $h\left(M_{t}\right)$ will eventually be greater than $h\left(M_{t-1}\right)$, and linear independence will follow. Thus $a \leqslant \beta-\delta$, and $\alpha-\gamma \leqslant \beta-\delta$.

A construction analogous to the above is used to derive the opposite inequality. Let $H \in A(U)[X]_{d}$ with $H+m A(U)[X] \in(k(U) \cdot \tau A f: \Theta-U I)$. Let $m(H)$ be the maximal integer $m$ such that there exists a form $M$ of degree $m$ and forms $H_{i}$ of degree $i=d+1, \ldots, m-1$ such that

$$
(\Theta-U \Pi)\left(H+H_{d+1}+\cdots+H_{m-1}\right)+M \in \omega A(U) f+A(U)[X]_{(m+1)}
$$

and $M+m A(U)[X] \notin(\tau A f, \Theta-u \Pi)$. If such a maximum does not exist then $H+m A(U)[X] \in H$, and if $H+m A(U)[X] \notin H$, then $m(H) \geqslant \operatorname{deg} H+1$. Let $M(H)$ be the set of all such $M$ of degree $m(H) . M(b H)=b M(H)$ for $b \in A(U) \sim$ $m A(U)$. If $M \in M(H)$ then $M+m A(U)[X] \subset M(H)$,

$$
M+m A(U)[X]_{m(H)}+(\tau A f, \Theta-U \Pi)_{m(H)} \subset M(H) / m A(U)[X]_{m(H)}
$$

and $M+m A(U)[X]_{m(H)} \in \tau(f, \Theta-U I) . M(H)$ will be considered as a subset of $\tau(f, \Theta-U \Pi) /(\tau A f, \Theta-U \Pi)$.

Let $H_{1}, \ldots, H_{\beta-\delta}$ be forms in $m A(U)[X]$ such that their residues modulo $m A(U)[X]$ are in $(k(U) \cdot \tau A f: \Theta-U I)$, such that their residues form a $k(U)$ basis for $(k(U) \cdot \tau A f: \Theta-U I) / H, m\left(H_{i}\right) \leqslant m\left(H_{i+1}\right)$ and such that if $m\left(H_{i}\right)=$ 
$m\left(H_{i+1}\right)$ then $\operatorname{deg} H_{i} \geqslant \operatorname{deg} H_{i+1}$. Choose $\mu_{i} \in M\left(H_{i}\right)$. Suppose $\mu_{1}, \ldots, \mu_{t-1}$ are linearly independent over $k(U)$ and $\mu_{t}=\bar{\alpha}_{1} \mu_{1}+\cdots+\bar{\alpha}_{t-1} \mu_{t-1}$ where $\alpha_{i} \in A(U) . \bar{\alpha}_{i}$ is nonzero only if $m\left(H_{i}\right)=m\left(H_{t}\right), m\left(H_{t-1}\right)=m\left(H_{t}\right)$ for $\mu_{t} \neq 0$, and let $H_{s}, \ldots, H_{t-1}$ be those $H_{i}$ with $i<t, m\left(H_{i}\right)=m\left(H_{t}\right)$ and $\operatorname{deg} H_{i}=$ $\operatorname{deg} H_{t}$. Then $m\left(H_{t}-\alpha_{s} H_{s}-\cdots-\alpha_{t-1} H_{t-1}\right)>m\left(H_{t}\right)$. Replace $H_{t}$ by $H_{t}-$ $\alpha_{s} H_{s}-\cdots-\alpha_{t-1} H_{t-1}$, choose $\mu_{t}$ anew, reorder $H_{1}, \ldots, H_{\beta-\delta}$, with a finite number of repetitions the injection is defined, and $\alpha-\gamma \geqslant \beta-\delta$.

Thus $\alpha-\gamma=\beta-\delta$. The final goal in the proof of $\alpha=\beta$ is to show that $\gamma$ and $\delta$ are equal.

Let $थ \subset B$ be two ideals of $A(U)$. As either $k(U)$ or $A(U)$-modules, $\tau \Re / \tau \Re$ $\simeq \sigma B / \sigma 2$. Now

$$
\begin{aligned}
& \sigma B / \sigma \mathscr{Z} \simeq \sum_{n>0} \oplus \frac{\left(m^{n} \cap \mathscr{Q}+m^{n+1} / m^{n+1}\right)}{\left(m^{n} \cap \mathscr{Q}+m^{n+1} / m^{n+1}\right)} \\
& \simeq \sum_{n \geq 0} \oplus \frac{\left(m^{n} \cap \mathscr{B}+m^{n+1}\right)}{\left(m^{n} \cap \mathscr{U}+m^{n+1}\right)} \simeq \sum_{n \geq 0} \bigoplus \frac{m^{n} \cap \mathscr{Z}}{\left(m^{n} \cap \mathscr{U}+m^{n+1} \cap \mathscr{Q}\right)}
\end{aligned}
$$

$\left(\right.$ for $\left.\left(m^{n} \cap \mathscr{B}\right) \cap\left(m^{n} \cap \mathscr{U}+m^{n+1}\right)=m^{n} \cap \mathscr{U}+m^{n+1} \cap \Re\right)$. Hence, $l_{k(u)} \tau \Re / \tau \mathscr{Q}=l_{A(U)} B / \mathfrak{Z T}$.

So

$$
\gamma=l_{A(U)}(P, f) /(y-x U, f),
$$

and

$$
\delta=l_{A(U)}(A(U) f: y-x U) / A(U) f .
$$

Let $\psi \in(A(U) f: y-x U) .(\psi / f)(y-x U) \in A(U), f(\psi / f)(y-x U) \in P$, $f \notin P$, so $(\psi / f)(y-x U) \in P$. Let $\xi_{1}(\psi)=(\psi / f)(y-x U)$. If $\psi \in A(U) f$ then $\xi_{1}(\psi) \in A(U)(y-x U)$. Hence

$$
\xi_{1}:(A(U) f: y-x U) / A(U) f \rightarrow(P, f) /(y-x U, f)
$$

is a homomorphism. Let $\psi \in \operatorname{Ker} \xi_{1}$, that is, let $(\psi / f)(y-x U)=a f+b(y-x U)$ for some $a$ and $b$ in $A(U)$. Then $(\psi-b f)(y-x U)=a f^{2}$, and $\psi \in\left(\left(A(U) f^{2}\right.\right.$ : $y-x U), f)$. If $\phi \in\left(A(U) f^{2}: y-x U\right)$, then $\phi(y-x U)=a f^{2}$ for some $a \in$ $A(U), \xi_{1}(\phi)=(\phi / f)(y-x U)=a f$, and $\phi \in \operatorname{Ker} \xi_{1}$. So

$$
\left.\operatorname{Ker} \xi_{1}=\left(A(U) f^{2}: y-x U\right), f\right) / A(U) f .
$$

Now,

$$
\begin{aligned}
\left(A(U) f^{i}: y-x U\right) /\left(A(U) f^{i}: y-x U\right) \cap A(U) f \\
\simeq\left(\left(A(U) f^{i}: y-x U\right), f\right) / A(U) f,
\end{aligned}
$$

and a homomorphism 


$$
\begin{aligned}
\xi_{i}:\left(A(U) f^{i}: y-x U\right) /\left(A(U) f^{i}: y-x U\right) \cap A(U) f \\
\quad \rightarrow\left(\cdots\left(((P, f) /(y-x U, f)) / \operatorname{Im} \xi_{1}\right) / \ldots\right) / \operatorname{Im} \xi_{i-1}
\end{aligned}
$$

with

$$
\operatorname{Ker} \xi_{i}=\left(\left(A(U) f^{i-1}: y-x U\right), f\right) / A(U) f
$$

is to be defined inductively.

If $\psi \in\left(A(U) f^{i}: y-x U\right)$, let $\xi_{i}(\psi)=\left(\psi / f^{i}\right)(y-x U) \in P$. If $\psi \in$ $\left(A(U) f^{i}: y-x U\right) \cap A(U) f$, then $\psi / f \in\left(A(U) f^{i-1}: y-x U\right), \xi_{i-1}(\psi / f)=$ $\left(\psi / f^{i}\right)(y-x U)=\xi_{i}(\psi)$, and $\xi_{i}(\psi) \in \operatorname{Im} \xi_{i-1}$. Let $\psi \in \operatorname{Ker} \xi_{i}$. Then

$\left(\psi / f^{l}\right)(y-x U)=a f+b(y-x U)$

$$
+\left(\psi_{1} / f\right)(y-x U)+\cdots+\left(\psi_{i-1} / f^{i-1}\right)(y-x U)
$$

where $\psi_{j} \in\left(A(U) f^{j}: y-x U\right)$ for $j=1, \ldots, i-1$, and

$$
\left(\psi-b f^{i}-f^{i-1} \psi_{1}-\cdots-f \psi_{i-1}\right)(y-x U)=a f^{i+1},
$$

so $\operatorname{Ker} \xi_{i} \subset\left(\left(A(U) f^{i+1}: y-x U\right), f\right) / A(U) f$. If $\phi \in\left(A(U) f^{i+1}: y-x U\right)$ then $\xi_{i}(\phi)=\left(\phi / f^{i}\right)(y-x U) \in A(U) f$, and $\phi \in \operatorname{Ker} \xi_{i}$. Thus

$$
\operatorname{Ker} \xi_{i}=\left(\left(A(U) f^{i+1}: y-x U\right), f\right) / A(U) f .
$$

$\bigcap_{i} A(U) f^{i}=(0)$, so $\bigcap_{i}\left(A(U) f^{i+1}: y-x U\right)=(0)$, and by [3, Theorem 30.1, p. 103], $\bigcap_{i} \operatorname{Ker} \xi_{i} \subset \bigcap_{k}\left(A(U) f+m^{k}\right)=A(U) f$. Or by [5, Theorem 1, p. 365], because $y-x U$ is superficial of degree $1,\left(m^{i+1} A(U): y-x U\right)=m^{i}$ for all sufficiently large $i$, so $\bigcap_{i} \operatorname{Ker} \xi_{i} \subset \bigcap_{i}\left(A(U) f+m^{l}\right)=A(U) f$. If $\phi \in P$ there is an integer $s$ such that $f^{s} \phi \in A(U)(y-x U)$, for there is an integer $s$ such that $P \cap m^{s}=A(U)(y-x U) \cap m^{s}$. Then $\xi_{s}\left(f^{s} \phi /(y-x U)\right)=\phi$.

Let

$$
\mathfrak{Q}_{i}=\left(\left(A(U) f^{i}: y-x U\right), f\right)
$$

and let

$$
\mathbb{B}_{i}=\left(\left\{\left(\psi / f^{i}\right)(y-x U) \mid \psi \in\left(A(U) f^{i}: y-x U\right)\right\}, f\right) .
$$

Then $\bigcap_{i} \mathscr{U}_{i}=A(U) f$ and $\mathscr{U}_{t}=A(U) f$ for some $t \geqslant 1$, for $(A(U) f: y-x U) / A(U) f$ is of finite length. Hence

$$
\mathscr{थ}_{0}=(A(U) f: y-x U) \supset \mathfrak{R}_{1} \supset \cdots \supset \mathfrak{\vartheta}_{t}=A(U) f,
$$

and

$$
(y-x U, f)=\mathbb{B}_{0} \subset \mathbb{B}_{1} \subset \cdots \subset \mathbb{P}_{s}=(P, f)
$$

where $\mathfrak{R}_{i} / \mathbb{R}_{i+1} \simeq \mathfrak{B}_{i+1} / \mathbb{B}_{i}$ as $A(U)$-modules. Thus $\gamma=\delta$.

The above construction is inductive to dimension one. Let $B_{d}=A$ and 
$B_{d-1}=B$ where $d$ is again the dimension of $A$, let $\Theta_{d-1}=\Theta, y_{d-1}=y, u_{d-1}=$ $u$ and $L_{d-1}=\Theta-U \Pi$. $\Pi$ and $x=\Pi\left(x_{i}\right)$ remain fixed throughout the induction. Suppose $B_{j+1}$ has been defined with the required properties. Let $\Theta_{j}$ be a form of degree one in $A[X]$ such that $y_{j}=\Theta_{j}\left(x_{i}\right)$ is a superficial element of $B_{j+1}$ and of $B_{j+1} / B_{j+1} f, \Theta_{j}$ is not contained in any associated prime ideal of $\left(p_{i}, L_{d-1}\right.$, $\ldots, L_{j+1}$ ) other than possibly $I$ nor contained in any isolated prime ideal of $\left(p_{i}, L_{d-1}, \ldots, L_{j+1}, \Pi\right)$ for any isolated prime ideal $p_{i}$ of $\tau A 0$, and such that $y_{j}$ is contained in no associated prime ideal of $B_{j+1} x$ except possibly $m B_{j+1}$. The above arguments hold when $A$ is replaced by $B_{j+1}$ and $B$ is replaced by $B_{j}=$ $S^{-1} B_{j+1}\left[u_{j}\right]$ where $u_{j}=y_{j} / x$ and $S=B_{j+1}\left[u_{j}\right] \sim m B_{j+1}\left[u_{j}\right]$.

Let $B=B_{1} . \quad B$ is one dimensional, $B$ is local with maximal ideal $m B$, and $\mu_{A}(f)=\mu_{B}(f)$.

Let $\Re^{1}$ be $T^{-1} \Re$ where $T=\Re \sim\left(p_{1} \cup \cdots \cup p_{r}\right)$ and where $p_{1}, \ldots, p_{r}$ are the height one prime ideals of $\Re$. For every $i=1, \ldots, r$,

$$
\Re^{1} \mathfrak{p}_{i} \cap A\left[u_{d-1}, \ldots, u_{1}\right]=m\left[u_{d-1}, \ldots, u_{1}\right] .
$$

For let $z \in A\left[u_{d-1}, \ldots, U_{1}\right] \cap \Re^{1} p$ where $p$ denotes one of the $f_{i}$. Then $z \in A\left[u_{d-1}, \ldots, u_{1}\right] \cap p$. Let $p$ be the prime ideal corresponding to $p$ which is associated to $\tau A 0$, and let $F\left(\Theta_{d-1}, \ldots, \Theta_{1}, \Pi\right)$ be a form in $\Theta_{d-1}, \ldots, \Theta_{1}$ and $\Pi$ with coefficients in $A$ such that

$$
F\left(\Theta_{d-1}\left(x_{i} / x\right), \ldots, \Theta_{1}\left(x_{i} / x\right), \Pi\left(x_{i} / x\right)\right)=z .
$$

$A\left[u_{d-1}, \ldots, u_{1}\right] \subset \Re$, so $z \in p$ and by the correspondence between $p$ and $p$, $F\left(\Theta_{d-1}, \ldots, \Theta_{1}, \Pi\right)+m[X] \in p$. Suppose $F$ modulo $m, \bar{F}$, is nonzero. If $\bar{F}$ were a power of $\Pi$, then $\Pi \in p$ which is a contradiction. So there is an integer $j$ such that $d-1 \geqslant j \geqslant 1, \bar{F} \in k\left[\Theta_{d-1}, \ldots, \Theta_{j}, \Pi\right]$ and $\bar{F} \notin k\left[\Theta_{d-1}, \ldots, \Theta_{j+1}, \Pi\right]$. Then

$$
\bar{F}=\bar{G} \Pi^{e} \bmod \left(\Theta_{d-1}-\Pi, \ldots, \Theta_{j+1}-\Pi\right) \subset\left(p, L_{d-1}, \ldots, L_{j+1}, \Pi\right)
$$

for some form $\bar{G} \in k\left[\Theta_{j}, \Pi\right]$ which is not divisible by $\Pi$. Letting $s \geqslant 1$ be the degree of $\bar{G}, \Theta_{j}^{s} \in\left(p, L_{d-1}, \ldots, L_{j+1}, \Pi\right)$ which is a contradiction to the choice of $\Theta_{j}$. Hence $\bar{F}=0$, and $z \in m\left[u_{d-1}, \ldots, u_{1}\right]$.

$B$ is a ring of fractions of $A\left[u_{d-1}, \ldots, u_{1}\right]$ with $m\left[u_{d-1}, \ldots, u_{1}\right] \subset$ $m B \cap A\left[u_{d-1}, \ldots, u_{1}\right], m B$ is a prime ideal of height one of $B$, so $m B \cap$ $A\left[u_{d-1}, \ldots, u_{1}\right]$ must be of height one also, and

$$
m B \cap A\left[u_{d-1}, \ldots, u_{1}\right]=m\left[u_{d-1}, \ldots, u_{1}\right] .
$$

It follows that

and therefore $B \subset \Re^{1}$.

$$
B=A\left[u_{d-1}, \ldots, u_{1}\right]_{m\left[u_{d-1}, \ldots, u_{1}\right]},
$$


$\Re^{1}=\Re_{1} \cap \cdots \cap \Re_{r}$ is a finite integral extension of $B=B_{1}$. The proof is an adaptation of the proof of Theorem $10[5, p .371]$. Let $p_{1}, \ldots, p_{r}$ also denote the proper prime ideals $\Re^{1} p_{1}, \ldots, \Re^{1} p_{r}$ of $\Re^{1}$, let $m_{i}$ be integers such that $p_{1}^{m_{1}} \cdots p_{r}^{m_{r}} \subset \mathfrak{R}^{1} m$, and let $n_{0}=p_{1}^{m_{1}} \cdots p_{r}^{m_{r}}$. Then $m^{s} \subset$ $\left(\Re^{1} m\right)^{s}$ and $\left(\Re^{1} m\right)^{s t} \subset m^{s}$ where $t=\max \left\{m_{1}, \ldots, m_{r}\right\}$. Let $\hat{B}$ be the $m B$ adic completion of $B$, and let $\hat{\Re}$ be the $\Re^{1} m$-adic completion of $\Re^{1}$. $\hat{\Re}$ is a $\hat{B}$ module, $\hat{\Re}$ is the m-adic completion of $\Re^{1}, \bigcap_{n \geq 0} \mathrm{~m}^{n}=(0)$, and by [7, Corollary 2, p. 273], the $m B$-adic topology of $B$ is induced by the m-adic topology of $\mathfrak{R}^{1}$. It is clear that $\hat{\mathfrak{R}} / \hat{\mathfrak{R}} m=\mathfrak{R}^{1} / \mathfrak{R}^{1} m$.

$B\left[x_{1} / x, \ldots, x_{n} / x\right]$ is of dimension one [3, Theorem 33.2, p. 115], and $\Re^{1}$ is a ring of quotients of $B\left[x_{1} / x, \ldots, x_{n} / x\right] . \beta_{j} \cap B\left[x_{1} / x, \ldots, x_{n} / x\right]$ for $j=1, \ldots, r$ are distinct proper prime ideals of $B\left[x_{1} / x, \ldots, x_{n} / x\right]$. Let $p$ be a proper prime ideal of $B\left[x_{1} / x, \ldots, x_{n} / x\right] . B\left[x_{1} / x, \ldots, x_{n} / x\right]$ is a ring of fractions of $A\left[x_{1} / x, \ldots, x_{n} / x\right]$, so $p \cap A\left[x_{1} / x, \ldots, x_{n} / x\right]$ is a prime ideal of height one, therefore there is a prime ideal $p$ of $\Re^{1}$ such that $p \cap$ $A\left[x_{1} / x, \ldots, x_{n} / x\right]=p \cap A\left[x_{1} / x, \ldots, x_{n} / x\right]$, and $p \cap B\left[x_{1} / x, \ldots, x_{n} / x\right]=$ $p$. From the above assertions it is immediate that $\Re^{1}=B\left[x_{1} / x, \ldots, x_{n} / x\right]$.

Let $\theta_{j i}$ be the residue of $x_{i} / x$ modulo $p_{j} . \Re^{1} / p_{j}=k\left(\bar{u}_{1}, \ldots, \bar{u}_{d-1}\right)$ $\left[\theta_{j 1}, \ldots, \theta_{j n}\right]$ is a field, and $\theta_{j i}$ are algebraic over $k(\bar{u})=k\left(\bar{u}_{1}, \ldots, \bar{u}_{d-1}\right)$. By multiplying together the $m_{j}$ th power of a polynomial which modulo $k_{j}$ is the algebraic relation of $\theta_{j i}$ over $k(\bar{u})$ for $j=1, \ldots, r$, there is a relation

$$
\left(x_{i} / x\right)^{t}+\alpha_{t-1}\left(x_{i} / x\right)^{t-1}+\cdots+\alpha_{0} \in \Re^{1} m
$$

where $\alpha_{0}, \ldots, \alpha_{t-1} \in B$. Therefore $\Re^{1} / \Re^{1} m$ is a finite $B / m B$ module, and $\hat{\Re}$ is a finite $\hat{B}$ module [7, Corollary 2, p. 259]. So for every positive integer $s$ there is a relation

$$
\begin{aligned}
\left(x_{i} / x\right)^{s} \in\left[\hat{B}\left(x_{i} / x\right)^{t-1}\right. & \left.+\cdots+\hat{B}\left(x_{i} / x\right)+\hat{B}\right] \cap B \\
& =B\left(x_{i} / x\right)^{t-1}+\cdots+B\left(x_{i} / x\right)+B
\end{aligned}
$$

for the latter module is finitely generated over the Zariski ring $B$ and is therefore closed. $\mathfrak{H}^{1}$ is thus finite integral over $B$.

It is to be shown that $\left[\Re^{1} / \mathbb{P}_{s}: B / m B\right]=e_{I}\left(k[X] / p_{s}\right)$. From the choice of $\Theta_{j}$ it follows that $L_{j}$ is a superficial element of

$$
k\left(\bar{u}_{d-1}, \ldots, \bar{u}_{j}\right)[X] /\left(p_{s}, L_{d-1}, \ldots, L_{j+1}\right),
$$

for $\bar{u}_{j}$ is transcendental over $k\left(\bar{u}_{d-1}, \ldots, \bar{u}_{j+1}\right)$. The dimensions are greater than one, so

$$
e_{I}\left(k[X] / p_{s}\right)=e_{I}\left(k(\bar{u})[X] /\left(p_{s}, L_{d-1}, \ldots, L_{1}\right)\right),
$$


where $k(\bar{u})$ now denotes $k\left(\bar{u}_{d-1}, \ldots, \bar{u}_{1}\right)$. Let $M_{k}(X) \in A[X]$ for $k=1$, $\ldots, t$ be forms of degree $d_{k}$ such that the residues of $M_{1}\left(x_{i} / x\right), \ldots, M_{t}\left(x_{i} / x\right)$ modulo $p_{s}$ form a basis of $\Re^{1} / \mathfrak{P}_{s}$ over $k(\bar{u})=B / m B$. If $G$ is a form in $A[X]$ of degree $g \geqslant \max \left\{d_{1}, \ldots, d_{t}\right\}$, then

$$
G\left(\theta_{s i}\right)=\sum_{k=1, \ldots, t} \alpha_{k}\left(\Pi\left(\theta_{s i}\right)\right)^{g-d_{k}} M_{k}\left(\theta_{s i}\right)
$$

for some $\alpha_{1}, \ldots, \alpha_{t} \in k(\bar{u})$, for $\Pi\left(\theta_{s i}\right)=1$. Letting

$$
0 \rightarrow K \rightarrow k(\bar{u})\left[X_{1}, \ldots, X_{n}\right] \rightarrow k(\bar{u})\left[\theta_{s 1}, \ldots, \theta_{s n}\right] \rightarrow 0
$$

be the exact where $X_{i} \rightarrow \theta_{s i}, k(\bar{u})[X]_{g} / K_{g}$ is of dimension $t$ over $k(\bar{u})$ for $g \geqslant \max \left\{d_{1}, \ldots, d_{t}\right\} . K \supset\left(p_{s}, L_{d-1}, \ldots, L_{1}\right)$ by the correspondence between $p_{s}$ and $p_{s}$. Let $G \in K_{g}$. There is a unit $\beta$ in $k(\bar{u})$ such that $\beta G \in$ $k[\bar{u}][X]_{g}$, and there are $F_{j} \in k[\bar{u}][X]$ for $j=1, \ldots, d-1$ such that

$$
E^{\prime}=\Pi^{c} \beta G=\sum_{j=1, \ldots, d-1}\left(\Theta_{j}-\bar{u}_{j} \Pi\right) F_{j} \in k[X]_{g+c}
$$

where $c$ is the degree of $\bar{u}$ in $\beta G$. Let $E \in A[X]_{g+c}$ be a representative of $E^{\prime}$. $E\left(x_{i} \mid x\right) \in p_{s}$, so $E^{\prime} \in p_{s^{\circ}}$. Thus $\Pi^{c} G \in\left(p_{s^{\prime}}, L_{d-1}, \ldots, L_{1}\right)$. Inductively $\Pi$ is contained in no minimal prime ideal of $\left(p_{s}, L_{d-1}, \ldots, L_{j}\right)$. For let $P$ be such a minimal prime ideal and suppose $\Pi \in P$. Then $\Theta_{j} \in P$, and inductively by dimension, $P$ is a minimal prime ideal of $\left(p_{s}, L_{d-1}, \ldots, L_{j+1}, \Pi\right)$ which is a contradiction to the choice of $\Theta_{j} .\left(p_{s}, L_{d}, \ldots, L_{1}\right)$ being of dimension one, $G$ is contained in every primary component of $\left(p_{s}, L_{d}, \ldots, L_{1}\right)$ except perhaps the primary component belonging to $I, K_{g}=\left(p_{s}, L_{d}, \ldots, L_{1}\right)_{g}$ for all large enough values of $g$, and by comparison of the Hilbert polynomials, $t=$ $e_{I}\left(k[X] / p_{s}\right)$.

Apply the first part of the proof of Lemma 1 to $\Re^{1}$ over $B=B_{1}$, and obtain

$$
\mu_{A}(f)=\mu_{B}(f)=\sum_{i=1, \ldots, r} e_{I}\left(k[X] / p_{i}\right) \mu_{\Re_{i}}(f) .
$$

4. The valuation formula. Let $A$ be a local ring with maximal ideal $m$. For a definition of a valuation of $A$, finite on $A$ and centered at a prime ideal of $A$, see $[2, \S 1]$. By the additivity formula $\mu_{A}(f)=\Sigma_{p} \lambda_{p}(f) e_{m}(A / p)$ where the sum ranges over all prime ideals $p$ of $A$ which are of depth equal to the dimension of $A$. Assume that $A$ is nonimbedded. Then the prime ideals $p$ are all of height one, but they do not necessarily include all the prime ideals of height one. Then also $\lambda_{p}(A f)$ is a finite sum of finite rank one discrete valuations centered at $p$.

As an example, let $A$ be an entire factorial ring of dimension greater than 
one. Let $\left\{v_{l}\right\}_{t \in I}$ be the set of prime divisors of type one of $A$, and let $p_{l}$ be a prime element of $A$ with $v_{l}\left(p_{)}\right)=1$. Let $w_{1}$ and $w_{2}$ be two distinct prime divisors of $A$ centered at $m$, let $a_{\iota}=w_{1}\left(p_{\iota}\right)$ and $b_{\iota}=w_{2}\left(p_{\imath}\right)$, and then $w_{1}=$

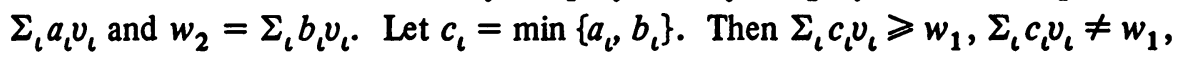
and $\Sigma_{l} c_{l} v_{l}$ is not a sum of valuations centered at $m$.

THEOREM. Let $A$ be a local ring with maximal ideal $m$. There are integral valued valuations $v_{1}, \ldots, v_{s}$ finite on $A$ centered at $m$, and there are positive integers $n_{1}, \ldots, n_{s}$ such that for every regular element $f$ of $A$,

$$
\mu_{A}(f)=n_{1} v_{1}(f)+\cdots+n_{s} v_{s}(f) .
$$

If $A$ is nonimbedded if $\mu_{A}(f)=n_{1} v_{1}(f)+\cdots+n_{s} v_{s}(f)$ for all regular elements $f$ of $A$, if the valuations $v_{1}, \ldots, v_{s}$ are independent, and if the ideal generated by each $v_{i}(A)$ is all of the integers, then the valuations $v_{1}, \ldots, v_{s}$ and the integers $n_{1}, \ldots, n_{s}$ are unique. (If $A$ is of dimension zero, $\mu_{A}$ is the trivial valuation: $\mu_{A}(f)=\infty$ if $f \in m$ and $\mu_{A}(f)=0$ if $f \notin m$.)

The proof of the formula is now straightforward. By Lemma 2, $A$ can be assumed to be entire. It may also be assumed that the residue field of $A$ is infinite. In fact let $A[x]$ be the polynomial ring in one variable over $A$, let $S=$ $A[x] \sim m A[x]$, and let $A(x)=S^{-1} A[x]$, a local ring with maximal ideal $m$. $A(x)$ and residue field $A(x) / m A(x)=k(x)$ a simple transcendental extension of $k=A / m$. Then $\mu_{A}=\mu_{A(x)}$, for $A(x) / A(x) f \simeq(A / A f)(x)$ and letting $B=A / A f$

$$
\begin{aligned}
G_{m B(x)} B(x) & =\sum_{n \geq 0} \frac{m^{n} B(x)}{m^{n+1} B(x)} \simeq \sum_{n \geq 0} \frac{m^{n}}{m^{n+1}} \otimes_{A} B(x) \\
& \simeq \sum_{n \geq 0} \frac{m^{n}+A f}{m^{n+1}+A f} \otimes_{k} k(x) \simeq\left(G_{m} B\right) \otimes_{k} k(x),
\end{aligned}
$$

so the multiplicities of $A / A f$ and of $A(x) / A(x) f$ are equal. A valuation of $A(x)$ restricted to $A$ remains a valuation. By Lemma $4, A$ can be assumed to be one dimensional, by Lemma $3, A$ can be assumed to be normal, and apply the Corollary of Proposition 2 to obtain the formula.

The proof of the unicity uses a slight generalization of the approximation theorem. Define two valuations of $\boldsymbol{A}$ to be equivalent if there is an order isomorphism and the usual commutative diagram, and to be independent if they are not equivalent.

Lemma. Let $Q$ be a noetherian nonimbedded ring which is its own total quotient ring. Let $v_{1}, \ldots, v_{s}$ be independent rank one valuations of $Q$, let $u_{1}, \ldots, u_{s} \in Q$ and let $\alpha_{i} \in v_{i}(A)$ be finite for $i=1, \ldots$, s. There is an element $u$ of $Q$ such that $v_{i}\left(u-u_{i}\right)=\alpha_{i}$ for $i=1, \ldots, s$. 
Proof. $Q=Q_{1} \oplus \cdots \oplus Q_{n}$ where $Q_{j}$ is a local ring of dimension zero, and let

$$
\Re_{j}=Q_{1} \oplus \cdots \oplus Q_{j-1} \oplus \Re_{j} \oplus Q_{j+1} \oplus \cdots \oplus Q_{n}
$$

where $\Re_{j}$ is the nil radical of $Q_{j}$. Let $v_{1}, \ldots, v_{t}$ be all of the valuations $v_{1}, \ldots, v_{s}$ which have $N_{v_{i}}=N_{1}$. Then $v_{1}, \ldots, v_{t}$ are naturally independent valuations of $Q / N_{1}=k_{1}$. By the approximation theorem for a field [7, Theorem 18, p. 45], there is an element $u_{1}^{\prime}$ of $Q_{1}$ with $v_{i}\left(u_{1}^{\prime}-\operatorname{proj}_{1} u_{i}\right)=\alpha_{i}$ for $i=1, \ldots, t$. Repeat this for each $N_{j}$, obtaining $u_{j}^{\prime} \in Q_{j}$ for $2 \leqslant j \leqslant n$. Let $u=u_{1}^{\prime} \oplus \cdots \oplus u_{n}^{\prime}$, and the proof of lemma is complete.

$A$ is assumed to be nonimbedded. Suppose $n_{1} v_{1}+\cdots+n_{s} v_{s} \geqslant 0$ where $v_{1}, \ldots, v_{s}$ are independent nontrivial rank one valuations finite on $A$. It is to be seen that $n_{1} \geqslant 0, \ldots, n_{s-1} \geqslant 0$ and $n_{s} \geqslant 0$. Let $u=f / g \in Q A$ where $f$ and $g$ are elements of $A$, such that for some $i, v_{i}(u)>0$ and $v_{j}(u)=0$ for $j \neq i$. Then $v_{i}(f)>v_{i}(g), v_{j}(f)=v_{j}(g)$ for $j \neq i, n_{i}\left(v_{i}(f)-v_{i}(g)\right) \geqslant 0$ and $n_{i} \geqslant 0$.

EXAMPLE. Let

$$
A=\mathrm{C}[x, y, z]_{(x, y, z)}=\mathrm{C}[X, Y, Z]_{(X, Y, Z)} /\left(X Y-Z^{3}\right)
$$

which is normal, analytically irreducible and Cohen-Macaulay. By direct computation $\mu_{A}(x)=\mu_{A}(y)=3, \mu_{A}(x+y)=2$, and $\mu_{A}$ is not a valuation. In fact, $\mu_{A}=$ $v_{x}+v_{y}$ where $\mathrm{C}(y / z)[z]_{(z)}$ and $\mathrm{C}(x / z)[z]_{(z)}$ are the valuation rings of $v_{x}$ and $v_{y}$ respectively. Note that neither $x$ nor $y$ are superficial elements of $A$.

EXAMPLE. Let

$$
A=k[w, x, y, z]_{(w, x, y, z)}=k\left[s^{4}, s^{3} t, s t^{3}, t^{4}\right]_{\left(s^{4}, s^{3} t, s t^{3}, t^{4}\right)} \subset k[s, t],
$$

the polynomial ring in two variables over a field $k . I A=k\left[s^{4}, s^{3} t, s^{2} t^{2}, s t^{3}, t^{4}\right]$, $D_{A}=\left\{\left(s^{4}, s^{3} t, s t^{3}, t^{4}\right)\right\}$ and $A$ is not Cohen-Macaulay. $A$ is the localization of a projective (graded) ring, and by Proposition 2, $\S 1, \mu_{A}=e_{m}(A) v_{A}$ where $v_{A}$ is the order valuation of $A$. By direct computation $\mu_{A}(x)=4$, so $e_{m}(A)=$ 4. Also $\Re=k(s / t)\left[t^{4}\right]_{\left(t^{4}\right)}$, which verifies the formula of the theorem for this example.

\section{REFERENCES}

1. J. Hornell, Intersection theory in an equicharacteristic regular local ring and the relative intersection theory, Proc. Amer. Math. Soc. 36 (1972), 8-12. MR 46\#9040.

2. - Divisorial complete intersections, Pacific J. Math. 45 (1973), 217-227. MR 47 \#6668.

3. M. Nagata, Local rings, Interscience Tracts in Pure and Appl. Math., no. 12, Interscience, New York, 1962. MR 27 \#5790.

4. D. G. Northcott, Lessons on rings, modules and multiplicities, Cambridge, Univ. Press, London, 1968. MR 38 \#144. 
5. D. G. Northcott, The neighborhoods of a local ring, J. London Math. Soc. 30 (1955), 360-375. MR 17, 86.

6. J.-P. Serre, Algèbre locale. Multiplicités, Lecture Notes in Math., vol. 11, Springer-Verlag, Berlin and New York, 1965. MR 34 \#1352.

7. O. Zariski and P. Samuel, Commutative algebra. Vol. Il, University Ser. in Higher Math., Van Nostrand, Princeton, N. J., 1960. MR 22 \#11006.

DEPARTMENT OF MATHEMATICS, UNIVERSITY OF KANSAS, LAWRENCE, KANSAS 66044

Current address: 2017 North 6th Street Terrace, Blue Springs, Missouri 64015 